BRAVZULIAN JOURNAL

OF MEDICAL AND BIOLOGICAL RESH.ARCH

www.bjournal.com.br
ISSN 0100-879X

Volume 44 (6) 497-605 June 2011

BIOMEDICAL SCIENCES

AND

CLINICAL INVESTIGATION

Braz J Med Biol Res, June 2011, Volume 44(6) 531-537

doi: 10.1590/S0100-879X2011007500060

\title{
An HPLC-UV method for the measurement of permeability of marker drugs in the Caco- 2 cell assay
}

J.M. Kratz, M.R. Teixeira, L.S. Koester and C.M.O. Simões

The Brazilian Journal of Medical and Biological Research is partially financed by

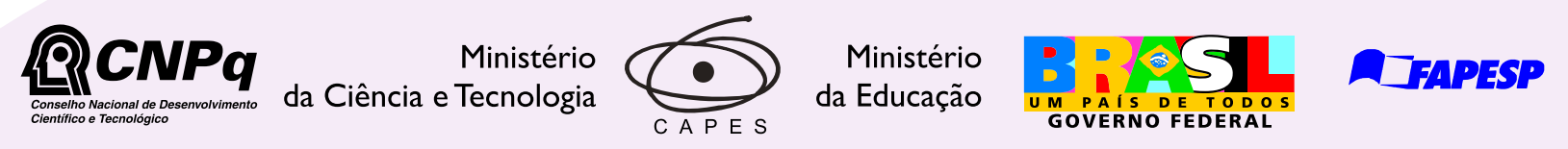

Institutional Sponsors
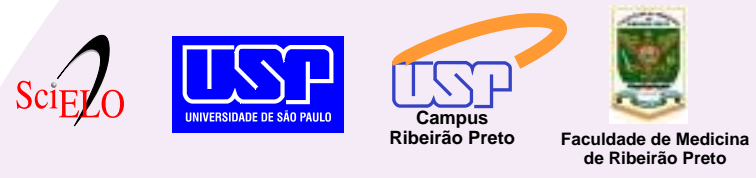
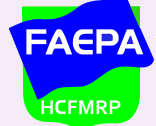

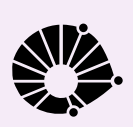

Ф SHIMADZU

GE Healthcare
Hotsite of proteomics metabolomics developped by:

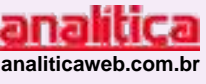

Thermo SCIENTIFIC 


\title{
An HPLC-UV method for the measurement of permeability of marker drugs in the Caco-2 cell assay
}

\author{
J.M. Kratz ${ }^{1}$, M.R. Teixeira ${ }^{1}$, L.S. Koester ${ }^{2}$ and C.M.O. Simões ${ }^{1}$ \\ ${ }^{1}$ Departamento de Ciências Farmacêuticas, Universidade Federal de Santa Catarina, \\ Florianópolis, SC, Brasil \\ ${ }^{2}$ Faculdade de Farmácia, Universidade Federal do Rio Grande do Sul, Porto Alegre, RS, Brasil
}

\begin{abstract}
The Caco-2 cell line has been used as a model to predict the in vitro permeability of the human intestinal barrier. The predictive potential of the assay relies on an appropriate in-house validation of the method. The objective of the present study was to develop a single HPLC-UV method for the identification and quantitation of marker drugs and to determine the suitability of the Caco-2 cell permeability assay. A simple chromatographic method was developed for the simultaneous determination of both passively (propranolol, carbamazepine, acyclovir, and hydrochlorothiazide) and actively transported drugs (vinblastine and verapamil). Separation was achieved on a C18 column with step-gradient elution (acetonitrile and aqueous solution of ammonium acetate, $\mathrm{pH} 3.0$ ) at a flow rate of $1.0 \mathrm{~mL} / \mathrm{min}$ and UV detection at $275 \mathrm{~nm}$ during the total run time of $35 \mathrm{~min}$. The method was validated and found to be specific, linear, precise, and accurate. This chromatographic system can be readily used on a routine basis and its utilization can be extended to other permeability models. The results obtained in the Caco- 2 bi-directional transport experiments confirmed the validity of the assay, given that high and low permeability profiles were identified, and Pglycoprotein functionality was established.
\end{abstract}

Key words: Caco-2; HPLC-UV; Marker drugs; Permeability

\section{Introduction}

Among the numerous techniques available for the prediction of intestinal permeability, the Caco-2 cell line has been extensively used and characterized as a model of the intestinal barrier $(1,2)$. These human cells are able to fully polarize into differentiated monolayers with well-established tight junctions and brush border membrane as well as to express several membrane transporters and metabolizing enzymes, allowing the measurement of functional permeability (both passive diffusion and active transport) (3). Consequently, this assay is widely accepted by both the pharmaceutical industry and regulatory agencies since the permeability determined using Caco-2 cells correlates well with oral absorption in humans (4-6).

In 2000, the United States Food and Drug Administration (FDA) published guidelines (7) based on the Biopharmaceutical Classification System (BCS) (8), whereby in vitro assays can be used to determine permeability class during the request of biowaivers of in vivo bioavailability and/or bioequivalence studies in immediate-release solid oral dosage forms.

More recently, the International Transporter Consortium (ITC), a group of industrial, regulatory and academic scientists with expertise in drug metabolism, transport and pharmacokinetics, presented their recommendations regarding the design of in vitro membrane transporter assays (9), particularly in relation to transporters involved in important clinical drug-drug interactions, highlighting the importance of these models in the early stages of the drug development process.

Although the Caco-2 assay presents many advantages, variable culture conditions can lead to inconsistent cell characteristics, poor reproducibility and relatively wide intra- and inter-laboratory variability. Therefore, cell culture techniques and experimental conditions should be specified in detail

Correspondence: C.M.O. Simões, Laboratório de Virologia Aplicada, Departamento de Ciências Farmacêuticas,

Universidade Federal de Santa Catarina, Campus Universitário Trindade, 88040-900 Florianópolis, SC, Brasil.

Fax: +55-48-3721-9258. E-mail: claudias@reitoria.ufsc.br

Received December 2, 2010. Accepted April 19, 2011. Available online May 13, 2011. Published June 13, 2011. 
$(10,11)$. The assay must be able to discriminate between high and low passive permeability profiles. Bi-directional transport experiments with $\mathrm{P}$-glycoprotein (P-gp) substrates and inhibitors are regarded as the definitive assay for the identification of active transport $(7,9)$. For the continuous assessment of the internal performance, marker drugs should be used during transport experiments $(7,12)$.

The objective of the present study was to develop an HPLC-UV method using a single chromatographic step for the determination of six marker drugs, acyclovir, carbamazepine, hydrochlorothiazide, propranolol, verapamil, and vinblastine, which were employed in the demonstration of the validity of the Caco- 2 permeability assay.

\section{Material and Methods}

\section{Chemicals and reagents}

The physicochemical properties and permeation mechanism of all the marker drugs used in this study are presented in Table 1. Acyclovir (ACV), carbamazepine (CBZ), hydrochlorothiazide (HTZ), and propranolol hydrochloride (PRO) reference standards ( $\geq 99 \%$ ) were obtained from the National Institute of Health Quality Control (INCQS, Brazil). Verapamil hydrochloride (VER, $\geq 99 \%$ ), vinblastine sulfate (VIN, $\geq 96 \%$ ), Hank's balanced salt solution (HBSS), sodium 4-(2-hydroxyethyl)-1-piperazineethanesulfonate (HEPES), methanesulfonic acid (MES), ethylenediaminetetraacetic acid (EDTA), trypsin, bovine serum albumin (BSA), dimethyl sulfoxide (DMSO), and Lucifer yellow (LY) were obtained from Sigma (USA). Acetonitrile and ammonium acetate (HPLC grade) were obtained from Tedia Co. (USA). Dulbecco's modified Eagle's medium (DMEM) with high glucose, fetal bovine serum (FBS), nonessential amino acids, and antibiotics/antimycotics were purchased from Invitrogen, Ltd. (USA). All other chemicals and reagents used in this study were of the highest commercially available purity.

\section{HPLC and chromatographic conditions}

Quantitative determinations of all six marker drugs were performed on a Perkin Elmer Series 200 HPLC instrument (USA), which consisted of a quaternary pump, vacuum degasser, autosampler, and diode-array detector (DAD). Separation was achieved on a C18 column (5 $\mu \mathrm{m}, 4.6 \mathrm{~mm}$ x 300 mm; Luna, Phenomenex, USA). A step-gradient elution was employed with acetonitrile as solvent $A$ and an aqueous solution of ammonium acetate, $\mathrm{pH} 3.0(25 \mathrm{mM})$, as solvent $B$, at a flow rate of $1.0 \mathrm{~mL} /$ min. Elution started at 3:97 (A:B, v/v) isocratic for $8.0 \mathrm{~min}$ and the gradient was then increased to $80: 20(A: B, v / v)$ for $14 \mathrm{~min}$, before being returned to the initial condition of 3:97 (A:B, v/v) for $8.0 \mathrm{~min}$, followed by $5 \mathrm{~min}$ equilibration. Effluent absorbance was measured at $275 \mathrm{~nm}$ and $20-\mu \mathrm{L}$ samples were injected into the column. UV spectra from 200 to $400 \mathrm{~nm}$ were recorded with the DAD online during the chromatographic run.

\section{Validation of the chromatographic method}

Method validation was performed based on both FDA and International Conference on Harmonization (ICH) guidelines for the validation of analytical methods $(13,14)$. All samples used during validation were freshly prepared in HBSS buffer. Exploration and optimization of several chromatographic parameters, such as selectivity ( $\alpha$ ), tailing factor $\left(T_{\mathrm{f}}\right)$, number of theoretical plates $(\mathrm{N})$ and peak resolution was performed, and the chromatogram showing the optimized separation of all six marker drugs is presented in Figure 1. Specificity was assessed by injecting individual drugs and recording their retention times and UV spectra. Chromatograms were compared to those of blank samples. Linearity was established by the construction of individual seven-point calibration curves over a range of $0.5-100 \mu \mathrm{M}$. Precision was established by both intra-day and inter-day precision. The intra-day precision was assessed by nine determinations covering the specified range (3 concentrations/3 replicates each), while the inter-day precision was assessed on different days by nine determinations covering the specified range (3 concentrations/3 replicates each/3 days). The relative standard deviation (RSD) of each drug was calculated as a measure of precision. The accuracy of the method was established by nine determinations covering the specified range (3 concentrations/3 replicates each 3 days). The percent difference of the mean values from the nominal concentrations was the measure of accuracy. The low limit of quantification (LLOQ) was determined as the lowest concentration in the calibration curve with a precision of $20 \%$ and accuracy of $80-120 \%$.

\section{Cell culture}

Caco-2 cells (HTB-37) were obtained from the American Type Culture Collection (ATCC, USA). Cells were maintained in a humidified $5 \% \mathrm{CO}_{2}$ air atmosphere at $37^{\circ} \mathrm{C}$ and were cultured in DMEM (4.5 g/L glucose) with $20 \%$ FBS, $1 \%$ non-essential amino acids, $100 \mathrm{U} / \mathrm{mL}$ penicillin, $100 \mu \mathrm{g} / \mathrm{mL}$ streptomycin, and $25 \mu \mathrm{g} / \mathrm{mL}$ amphotericin B. After reaching $80-90 \%$ confluence, cells were harvested with $0.25 \%$ trypsin/ EDTA solution, and counted by the Trypan blue exclusion method on a Countess ${ }^{\circledR}$ automated cell counter (Invitrogen). Only cultures with $\geq 95 \%$ viability were used.

\section{Transport studies}

For the intestinal permeability experiments, Caco-2 cells between passages 25 and 31 were seeded on Millicell ${ }^{\circledR}$ polycarbonate inserts $\left(0.6 \mathrm{~cm}^{2}, 0.4 \mu \mathrm{m}\right.$ pore size; Millipore, USA) at a density of 100,000 cells per insert and cultivated for 21-25 days. Culture medium was replaced three times per week until the time of use.

The transepithelial electrical resistance (TEER) of the monolayers and the permeability to the paracellular marker LY were considered to be indicators of monolayer integrity. TEER was assessed at $37^{\circ} \mathrm{C}$ using a Millicell ${ }^{\circledR}$ ERS meter (Millipore) connected to a WPI ${ }^{\circledR}$ Endohm tissue resistance 
measurement chamber (USA) and reported as $\Omega \mathrm{cm}^{2}$. The permeability of the monolayers to LY $(100 \mu \mathrm{g} / \mathrm{mL})$ was assessed using a Tecan ${ }^{\circledR}$ Infinite 200 microplate reader (Switzerland). Only monolayers with TEER values above 200 $\Omega \mathrm{cm}^{2}$ and LY permeability $\leq 0.2 \mathrm{x}$ $10^{-6} \mathrm{~cm} / \mathrm{s}$ were used.

The transport experiments were carried out according to the recommendations previously described (15). HBSS at $\mathrm{pH} 6.0$ (10 mM MES) and $\mathrm{pH} 7.4$ (10 $\mathrm{mM}$ HEPES) was used as transport buffer in the apical (AP) and basolateral (BL) compartments, respectively, in order to mimic in vivo conditions. Before the experiments, monolayers were washed twice with HBSS, $\mathrm{pH} \mathrm{7.4,} \mathrm{and}$ incubated for $30 \mathrm{~min}$ at $37^{\circ} \mathrm{C}$ for TEER measurement. Next, DMSO stock solutions ( $1 \mathrm{mM}$ ) of all marker drugs were diluted in HBSS and added to the donor compartment, while fresh HBSS was added to the receiver compartment.

The permeability of passive diffusion markers was assessed on isolated drugs (PRO, CBZ, $A C V$, and $H T Z$ ) or on a drug mixture (CBZ and HTZ). The $\mathrm{P}$-gp functionality was evaluated with VIN alone (P-gp substrate) or in the presence of VER (P-gp inhibitor; 100 $\mu \mathrm{M}$; both compartments). All experiments were performed in both directions, i.e., from $A P$ to $B L$ and from $B L$ to $A P$, for the period of $2 \mathrm{~h}$, at $37^{\circ} \mathrm{C}$ in an orbital shaker (100 rpm). At appropriate times, $100-\mu \mathrm{L}$ aliquots were removed from the receiver compartment and replaced with an equal volume of fresh HBSS. At the end of the experiment, samples were collected from donor compartments in order to perform the mass balance calculation.

Transport experiments were conducted under sink conditions (where less than $10 \%$ of the drug was transported across the cell monolayer) and the apparent permeability coefficient $\left(P_{\text {app }}\right)(\mathrm{cm} / \mathrm{s})$ values were calculated as follows (15):

$P_{a p p}=\frac{(\Delta \mathrm{Q})}{(\Delta \mathrm{t})} \times \frac{1}{A \times C_{0} \times 60}$

Eq. 1

where $(\Delta Q / \Delta t)$ is the steady-state flux $(\mathrm{mol} / \mathrm{s}), A$ is the surface area of the filter $\left(\mathrm{cm}^{2}\right)$ and $C_{0}$ is the initial concentration in the donor compartment $(\mathrm{mol} / \mathrm{mL})(16)$. For rapidly transported drugs, where sink conditions could not be maintained for the full duration of the experiments, permeability coefficient $\left(P_{\text {app }}\right)$ values $(\mathrm{cm} / \mathrm{s})$ were calculated as follows:

$$
C_{R}(t)=\frac{M}{V_{D}+V_{R}}+\left(C_{R, 0}-\frac{M}{V_{D}+V_{R}}\right) e^{-P_{a p p} A\left(1 / V_{D}+V_{R}\right) t}
$$

Eq. 2

where $C_{R}(t)$ is the time-dependent drug concentration in the receiver compartment, $M$ is the amount of drug in the system, $V_{D}$ and $V_{R}$ are the volumes of the donor and receiver compartments, respectively, and $t$ is the time from the start of the interval. $P_{\text {app }}$ values were obtained from nonlinear regression minimizing the sum of squared residuals $\left(\sum\left(C_{\mathrm{R}, \mathrm{i}, \mathrm{obs}}-C_{\mathrm{R}, \mathrm{i}, \mathrm{calc}}\right)^{2}\right)$, where $C_{\mathrm{R}, \mathrm{i}, \mathrm{obs}}$ is the observed receiver concentration at the end of the interval and $C_{R, i, c a l c}$ is the corresponding concentration calculated according to Equation $2(16,17)$.

\section{Statistical analysis}

Data are reported as the mean \pm SD of three independent experiments. For transport experiments, triplicate 
inserts were used in each experiment repetition. Microsoft Excel $2007^{\circledR}$ (USA) was used for the linear and non-linear regression analyses.

\section{Results and Discussion}

The Caco-2 cell model is the most frequently used method for in vitro gastrointestinal permeability assessment. The experience collected with this cell line over the last two decades has shown both successes and failures in its predictive capability (18). From this standpoint, the appropriate validation of the assay within research laboratories is mandatory for the suitable application of the assay to drug profiling projects in order to avoid the intrinsic variability of cell culture techniques. Therefore, the main purpose of the present study was to determine the suitability of a Caco-2 assay through the determination of marker drugs.

\section{Marker drug set assembly}

Six marker drugs with known fraction absorbed in humans (FA\%) and a broad range of physicochemical properties were selected based on published data (Table 1) (17,19-23). Drugs were divided into two experimental groups: one for the determination of passive permeability, and the other for the demonstration of P-gp functionality. In agreement with FDA recommendations (7), these drugs encompass a wide range of FA\% (17-100\%) covering all four BCS classes. For the measurement of functional efflux mediated by P-gp, VIN and VER were selected as substrate and inhibitor, respectively, since both drugs have already been described as model drugs for cellular P-gp interaction studies (9).

\section{HPLC}

The optimum wavelength for the detection of all analytes with adequate sensitivity and specificity was found to be $275 \mathrm{~nm}$. A fixed UV wavelength was used in order to allow equipment interchangeability, even though DAD was available. The next step was the selection of the mobile phase components and composition. Acetonitrile (solvent A) and ammonium acetate (25 mM; solvent $B$ ) were selected for the initial studies. In order to achieve a balance between adequate peak resolution and total run time, a gradient elution was programmed. Best peak shapes were obtained with $\mathrm{pH} 3.0$ in the aqueous phase with a flow rate of 1.0 $\mathrm{mL} / \mathrm{min}$, since lower flows improved tailing, while a higher flow generated elevated backpressure.

ACV was initially challenging, since this analyte required a high aqueous content ( $3: 97, \mathrm{v} / \mathrm{v})$ for adequate peak shape and retention time. The remaining drugs were eluted by a gradual shift to a high organic content (80:20, v/v). With these conditions, only PRO and VIN presented low peak resolution ( $\leq 2.0)$. To improve this feature, a $300-\mathrm{mm} \mathrm{C18}$ column was employed in place of the standard $150-\mathrm{mm}$ column initially used. With these optimized chromatographic conditions, the average retention times were 8.88, 20.90, 23.95, 24.43, 26.22, and 26.58 min, for ACV, HTZ, PRO, VIN, VER, and CBZ, respectively (Figure 1).

Table 2 summarizes linearity, range, precision, and accuracy data. VER was not included in Table 2 since it was used exclusively for inhibition of P-gp. Although a baseline alteration due to gradient shift was detected at about $10 \mathrm{~min}$, no interference was observed in the retention time windows of each analyte, and specificity was confirmed by comparison of UV spectra to previously published data (24). The LLOQs were determined as $0.5 \mu \mathrm{M}$ for all drugs (lowest concentration in the calibration curve), since the RSDs at this concentration were $\leq 1.84 \%$. The data in Table 2 show that the method presented here is appropriate for the determination of these markers $(13,14)$.

This method can be readily employed for the suitability demonstration and in-house validation of Caco-2 permeability assays. In addition, the applicability of this method can be extended to other in vitro/in situ/ex vivo permeability assays. Although several studies have reported the simultaneous determination of permeability of marker drugs by HPLC (25-29), to our knowledge, this is the first report of an HPLC-UV method for the determination of the $\mathrm{P}$-gp substrate VIN concomitantly with the corresponding inhibitor VER, and the four passive permeability markers used in this study.

Table 1. Physicochemical properties of marker drugs used in this study.

\begin{tabular}{lcccccc}
\hline Marker drug & Permeability class & BCS class & Transport mechanism & FA\% & pKa & $\log P$ \\
\hline Propranolol & High & I & Transcellular & $90 \%$ & 9.52 & 3.43 \\
Carbamazepine & High & II & Transcellular & $100 \%$ & - & 2.45 \\
Acyclovir & Low & III & Paracellular & $17 \%$ & $2.34,9.23$ & -2.42 \\
Hydrochlorothiazide & Low & IV & Paracellular & $55 \%$ & $8.77,9.79$ & -0.17 \\
Vinblastine & - & - & P-gp substrate & - & 7.4 & 4.32 \\
Verapamil & High & I & P-gp inhibitor & $98 \%$ & 8.66 & 3.96 \\
\hline
\end{tabular}

BCS = Biopharmaceutical Classification System (8); FA\% = fraction absorbed in humans; $\log P=$ partition coefficient; P-gp = P-glycoprotein. The data presented in this table were obtained from Refs. 17,19-23. 
Caco-2 permeability assay - suitability demonstration

The formation of a functional single monolayer on polycarbonate inserts is an important feature regarding the mimicking of in vivo conditions in the Caco-2 permeability assay. As small changes in the culture conditions can lead to significant differences in cell phenotype (10), the integrity of the monolayers and the formation of a robust tight junction (TJ) network were monitored by TEER measurement and LY transport.

TEER profiles were obtained throughout the period of culture (21-25 days). Detectable values emerged from the 4th day of culture (values around $100 \Omega \mathrm{cm}^{2}$ ) and continued to increase, until reaching a plateau on the 11th day of culture. The average TEER value obtained before the transport experiments was $297 \pm 21 \Omega \mathrm{cm}^{2}$. Additional information regarding the integrity and tightness of monolayers was obtained by LY permeability assays. LY is a fluorescent paracellular marker with very low permeability and has been used as a marker of TJ maturation (30). The average LY $P_{\text {app }}$ value obtained after 21 days of culture was $\leq 0.2 \mathrm{x}$ $10^{-6} \mathrm{~cm} / \mathrm{s}$, in agreement with previously published data (31). For cut-off purposes, only monolayers with TEER values above $200 \Omega \mathrm{cm}^{2}$ and LY permeability $\leq 0.2 \times 10^{-6} \mathrm{~cm} / \mathrm{s}$ were considered to be appropriate for the experiments.

Through bi-directional transport experiments, $P_{\text {app }}$ values were determined for each marker drug across Caco-2 cell monolayers and the summary of the permeability data is shown in Table 3. Four marker drugs were employed for passive permeability evaluation, i.e., PRO and CBZ as high permeability standards, along with ACV and HTZ as low permeability standards. All drugs presented adequate $P_{\text {app }}$ values, which correlated well with the FA\% of these drugs, allowing the establishment of a rank order relationship as intended.

Another important aspect considered was the efflux ratios ( $P_{\text {app BL-AP }} / P_{\text {app AP-BL}}$ ) obtained. Passively transported drugs should produce efflux ratios close to 1.00 in order to demonstrate independence from active transport mechanisms (9). In the present study, only CBZ, ACV and HTZ presented proper values, while PRO showed an efflux ratio of 2.81, which could lead to a false interpretation toward an active transport pathway. However, since a $\mathrm{pH}$ gradient setup was employed in the transport experiments, and $\mathrm{PRO}$ is a basic drug with $\mathrm{p} K_{\mathrm{a}}$ around 9.5 , data analysis revealed that $\mathrm{PRO}$ permeability was $\mathrm{pH}$-sensitive due to ionization effects, as already described (30).

Moreover, the permeability of CBZ and HTZ was assessed via a cocktail approach. The results obtained with this strategy were very similar, and maintained the rank order agreement. CBZ presented AP-BL and BL-AP $P_{\text {app }}(\mathrm{cm} / \mathrm{s})$ values of $110.61 \pm 3.41$ and $116.6 \pm 5.50$, while HTZ presented values of $1.81 \pm 0.22$ and $2.36 \pm 0.34$, respectively. Therefore, CBZ and HTZ were selected to be used as a single mixture control for the continuous evaluation of the internal performance of the Caco-2 permeability assay.

The permeability profile of VIN, a P-gp substrate, was also determined in order to demonstrate the suitability of the method regarding active transport mechanisms. Special attention was given to the selection of drug concentration in order to avoid transporter saturation $(9,32)$. As shown

Table 2. Chromatographic validation data for the determination of marker drugs used in the Caco-2 permeability assay.

\begin{tabular}{|c|c|c|c|c|c|c|c|}
\hline \multirow[t]{2}{*}{ Marker drug } & \multirow{2}{*}{$\begin{array}{l}\text { Range } \\
(\mu \mathrm{M})\end{array}$} & \multirow[t]{2}{*}{ Equation $\left(r^{2}\right)$} & \multirow{2}{*}{$\begin{array}{c}\text { Theoretical } \\
\text { concentration } \\
(\mu \mathrm{M})\end{array}$} & \multicolumn{2}{|c|}{ Intra-day } & \multicolumn{2}{|c|}{ Inter-day } \\
\hline & & & & Accuracy (\%) & $\%$ RSD & Accuracy (\%) & $\%$ RSD \\
\hline \multirow[t]{3}{*}{ Propranolol } & \multirow[t]{3}{*}{$0.5-100$} & \multirow{3}{*}{$\begin{array}{c}y=7652 x-7957 \\
(0.9996)\end{array}$} & 50 & 103.61 & 0.56 & 97.24 & 3.55 \\
\hline & & & 25 & 105.13 & 0.71 & 89.06 & 3.38 \\
\hline & & & 10 & 111.68 & 0.40 & 96.50 & 0.23 \\
\hline \multirow[t]{3}{*}{ Carbamazepine } & \multirow[t]{3}{*}{$0.5-100$} & \multirow{3}{*}{$\begin{array}{c}y=24003 x-16365 \\
(0.9997)\end{array}$} & 50 & 99.48 & 0.68 & 91.48 & 1.22 \\
\hline & & & 25 & 101.30 & 0.31 & 92.20 & 3.72 \\
\hline & & & 10 & 103.75 & 0.35 & 98.31 & 1.50 \\
\hline \multirow[t]{3}{*}{ Acyclovir } & \multirow[t]{3}{*}{$0.5-100$} & \multirow{3}{*}{$\begin{array}{c}y=15244 x-7135 \\
(0.9999)\end{array}$} & 50 & 109.40 & 1.96 & 93.03 & 9.55 \\
\hline & & & 25 & 113.22 & 2.86 & 93.34 & 10.41 \\
\hline & & & 10 & 115.37 & 1.42 & 95.46 & 10.53 \\
\hline \multirow[t]{3}{*}{ Hydrochlorothiazide } & \multirow[t]{3}{*}{$0.5-100$} & \multirow{3}{*}{$\begin{array}{c}y=36301 x-7957 \\
(0.9998)\end{array}$} & 50 & 96.23 & 0.21 & 92.82 & 1.05 \\
\hline & & & 25 & 97.52 & 0.60 & 96.58 & 3.49 \\
\hline & & & 10 & 98.48 & 0.10 & 90.99 & 0.95 \\
\hline \multirow[t]{3}{*}{ Vinblastine } & \multirow[t]{3}{*}{$0.5-100$} & \multirow{3}{*}{$\begin{array}{c}y=16664 x-13615 \\
(0.9991)\end{array}$} & 50 & 111.12 & 0.86 & 100.11 & 1.78 \\
\hline & & & 25 & 104.55 & 1.27 & 96.57 & 3.54 \\
\hline & & & 10 & 92.23 & 1.26 & 97.62 & 5.30 \\
\hline
\end{tabular}

Intra-day and inter-day accuracy and precision were determined in triplicate for each concentration. Accuracy was defined as percent difference from the nominal concentration. Precision is reported as the relative standard deviation (RSD). 
in Table 3, an efflux ratio of 17.75 was obtained. An acceptable system produces a minimum efflux ratio of 2.00 (9). Moreover, the permeability of VIN was evaluated in the presence of VER, a known P-gp inhibitor. In this setup, the $P_{\text {app AP-BL value }}$ obtained was 4.5-fold higher than that of VIN alone, and the efflux ratio observed was much lower (4.28). These findings agree with recommendations that the efflux ratio should be significantly reduced by the addition of a known inhibitor (more than $50 \%)(9,32)$, as observed in the present study, thereby corroborating the adequacy of the Caco-2 permeability assay in relation to P-gp functionality.

A simple, accurate and precise HPLC method using UV detection was developed and validated for the simultaneous determination of marker drugs directly in the transport buffer. This method was successfully employed in the Caco-2 permeability assay during the in-house validation of the technique within our research laboratory, and can be readily employed on a routine basis with other permeability models. The results obtained in the present study confirmed the suitability of the Caco-2 permeability assay for the assessment of high and low permeability profiles, and for the

\section{References}

1. Balimane PV, Han YH, Chong S. Current industrial practices of assessing permeability and P-glycoprotein interaction. AAPS J 2006; 8: E1-13.

2. Press B, Di Grandi D. Permeability for intestinal absorption: Caco-2 assay and related issues. Curr Drug Metab 2008; 9: 893-900.

3. Hidalgo IJ, Raub TJ, Borchardt RT. Characterization of the human colon carcinoma cell line (Caco-2) as a model system for intestinal epithelial permeability. Gastroenterology 1989; 96: 736-749.

4. Artursson P, Karlsson J. Correlation between oral drug absorption in humans and apparent drug permeability coefficients in human intestinal epithelial (Caco-2) cells. Biochem Biophys Res Commun 1991; 175: 880-885.

5. Elsby R, Surry DD, Smith VN, Gray AJ. Validation and application of Caco-2 assays for the in vitro evaluation of development candidate drugs as substrates or inhibitors of P-glycoprotein to support regulatory submissions. Xenobiotica 2008; 38: 1140-1164.

6. Matsson P, Bergstrom CA, Nagahara N, Tavelin S, Norinder $\mathrm{U}$, Artursson P. Exploring the role of different drug transport routes in permeability screening. $J$ Med Chem 2005; 48: establishment of P-gp functionality. To our knowledge, this is the first report of an HPLC-UV method developed for the determination of vinblastine concomitantly with verapamil, and four other passive permeability markers - propranolol, carbamazepine, acyclovir, and hydrochlorothiazide.

\section{Acknowledgments}

This article was dedicated to Universidade Federal de Santa Catarina on the occasion of the 50th year since the foundation of this institution. The authors are grateful to FAPESC (grant \#5780/2007-0), CAPES/MEC, and CNPq/ MCT for financial support and research scholarships.
604-613.

7. FDA (Food and Drug Administration). Guidance for industry - Waiver of in vivo bioavailability and bioequivalence studies for immediate-release solid oral dosage forms based on a biopharmaceutics classification system. 2000.

8. Amidon GL, Lennernas $\mathrm{H}$, Shah VP, Crison JR. A theoretical basis for a biopharmaceutic drug classification: the correlation of in vitro drug product dissolution and in vivo bioavailability. Pharm Res 1995; 12: 413-420.

9. Giacomini KM, Huang SM, Tweedie DJ, Benet LZ, Brouwer $\mathrm{KL}$, Chu X, et al. Membrane transporters in drug development. Nat Rev Drug Discov 2010; 9: 215-236.

10. Volpe DA. Variability in Caco-2 and MDCK cell-based intestinal permeability assays. J Pharm Sci 2008; 97: 712-725.

11. Corti G, Maestrelli F, Cirri M, Zerrouk N, Mura P. Development and evaluation of an in vitro method for prediction of human drug absorption II. Demonstration of the method suitability. Eur J Pharm Sci 2006; 27: 354-362.

12. Polli JE, Yu LX, Cook JA, Amidon GL, Borchardt RT, Burnside BA, et al. Summary workshop report: biopharmaceutics classification system - implementation challenges and extension opportunities. J Pharm Sci 2004; 93: 1375-1381. 
13. FDA (Food and Drug Administration). Guidance for industry - Bioanalytical method validation. 2001.

14. ICH (International Conference on Harmonization). Validation of analytical procedures: text and methodology, Q2(R1). 2005.

15. Hubatsch I, Ragnarsson EG, Artursson P. Determination of drug permeability and prediction of drug absorption in Caco2 monolayers. Nat Protoc 2007; 2: 2111-2119.

16. Grasjo J, Taipalensuu J, Ocklind G, Artursson P. Applications of epithelial cell culture in studies of drug transport. In: Wise C (Editor), Epithelial cell culture protocols. Totowa: Humana Press Inc.; 2002. p 233-272.

17. Bergstrom CA, Strafford M, Lazorova L, Avdeef A, Luthman $\mathrm{K}$, Artursson P. Absorption classification of oral drugs based on molecular surface properties. J Med Chem 2003; 46: 558-570.

18. Ungell AL. Caco-2 replace or refine? Drug Discovery Today 2004; 1: 423-430.

19. Winiwarter S, Bonham NM, Ax F, Hallberg A, Lennernas $\mathrm{H}$, Karlen A. Correlation of human jejunal permeability (in vivo) of drugs with experimentally and theoretically derived parameters. A multivariate data analysis approach. $J$ Med Chem 1998; 41: 4939-4949.

20. Lee KJ, Johnson N, Castelo J, Sinko PJ, Grass G, Holme K, et al. Effect of experimental $\mathrm{pH}$ on the in vitro permeability in intact rabbit intestines and Caco-2 monolayer. Eur J Pharm Sci 2005; 25: 193-200.

21. Wu CY, Benet LZ. Predicting drug disposition via application of BCS: transport/absorption/elimination interplay and development of a biopharmaceutics drug disposition classification system. Pharm Res 2005; 22: 11-23.

22. Skold C, Winiwarter S, Wernevik J, Bergstrom F, Engstrom $\mathrm{L}$, Allen $\mathrm{R}$, et al. Presentation of a structurally diverse and commercially available drug data set for correlation and benchmarking studies. J Med Chem 2006; 49: 6660-6671.

23. Ahlin G, Karlsson J, Pedersen JM, Gustavsson L, Larsson R, Matsson $\mathrm{P}$, et al. Structural requirements for drug inhibition of the liver specific human organic cation transport protein 1. J Med Chem 2008; 51: 5932-5942.

24. Moffat AC, Osselton MD, Widdop B (Editors). Clarke's analysis of drugs and poisons. Chicago: Pharmaceutical Press; 2003.

25. Bansal T, Singh M, Mishra G, Talegaonkar S, Khar RK, Jaggi $\mathrm{M}$, et al. Concurrent determination of topotecan and model permeability markers (atenolol, antipyrine, propranolol and furosemide) by reversed phase liquid chromatography: utility in Caco-2 intestinal absorption studies. J Chromatogr $B$ Analyt Technol Biomed Life Sci 2007; 859: 261-266.

26. Venkatesh G, Ramanathan S, Mansor SM, Nair NK, Sattar MA, Croft SL, et al. Development and validation of RPHPLC-UV method for simultaneous determination of buparvaquone, atenolol, propranolol, quinidine and verapamil: a tool for the standardization of rat in situ intestinal permeability studies. J Pharm Biomed Anal 2007; 43: 1546-1551.

27. Chawla S, Ghosh S, Sihorkar V, Nellore R, Kumar TR, Srinivas NR. High-performance liquid chromatography method development and validation for simultaneous determination of five model compounds, antipyrine, metoprolol, ketoprofen, furosemide and phenol red, as a tool for the standardization of rat in situ intestinal permeability studies using timed wavelength detection. Biomed Chromatogr 2006; 20: 349-357.

28. Augustijns P, Mols R. HPLC with programmed wavelength fluorescence detection for the simultaneous determination of marker compounds of integrity and P-gp functionality in the Caco-2 intestinal absorption model. J Pharm Biomed Anal 2004; 34: 971-978.

29. Palmgren JJ, Monkkonen J, Jukkola E, Niva S, Auriola S. Characterization of Caco-2 cell monolayer drug transport properties by cassette dosing using UV/fluorescence HPLC. Eur J Pharm Biopharm 2004; 57: 319-328.

30. Thiel-Demby VE, Humphreys JE, St John Williams LA, Ellens HM, Shah N, Ayrton AD, et al. Biopharmaceutics classification system: validation and learnings of an in vitro permeability assay. Mol Pharm 2009; 6: 11-18.

31. Uchida M, Fukazawa T, Yamazaki Y, Hashimoto H, Miyamoto Y. A modified fast (4 day) 96-well plate Caco-2 permeability assay. J Pharmacol Toxicol Methods 2009; 59: 39-43.

32. Shirasaka Y, Sakane T, Yamashita S. Effect of P-glycoprotein expression levels on the concentration-dependent permeability of drugs to the cell membrane. J Pharm Sci 2008; 97: 553-565. 